Doel. $-1, \ldots, T 3$

DOE/ER/51116--T3

DE92 003252

\title{
COOPERATIVE PROGRAM ON DIII-D
}

Principal Investigator: T. Kenneth Fowler Unir of Califumia, Berpelex

This is a proposal for a three-year grant (FY92-94) to continue collaboration with Gene:al Atornics and LLNL staff in analyzing data from the DIII-D tokamak facility and related activities. This is a continuation of work funded under U.S. Department of Energy Contract DE-FG03-89ER51116 that ends November 10, 1991. The primary focus is on plasma transport processes and their consequences, with increasing emphasis on edge physics.

Tasks included in the present proposal have been selected, in consultation with the DIII-D staff, to meet specific needs of the DIII-D program. Our work is now fully integrated into the ongoing efforts at GA to help expedite progress in the fundamental understanding of tiansport phenomena in tokamaks. Results to date, outlined below, have been reported in 24 journal publications, laboratory reports and meeting presentations listed herein. Close communication with GA staff members is maintained by computer linkage and by frequent trips to San Diego. Dr. Gary Porter, who leads the Livermore DIII-D team, is a regular participant in our group meetings in Berkeley.

\section{PROGRESS UNDER PRESENT GRANT}

The main contribution of the Berkeley group to date has concerned ion temperature profile data seduction and transport analysis using this data. In addition, our graduate students have worked on fundamental aspects of transport theory, under the guidance of the Principal Investigator, to prepare them for productive participation in the DIII-S program. One of these students, Q. Nguyen, has written a paper with Drs. Stambaugh and Fowler on divertor design, a subject of increasing urgency for ITER and an area of increasing importance in the DIII-D program. Finally, work has been completed $u n$ determining upper bounds on fluctuation levels and growth constants, relevant to core plasma transport calculations, using thermodynamic methods. The following is a brief summary of this work, with emphasis on the accomplishments during the past year.

\section{ION TEMPERATURE ANALYSIS}

Among the data essential for transport analysis in DIII-D, the ion temperature profile analysis has proven to be most difficult to a'tomate, and in fact this analysis has generally required the time-consuming attention of the experimentalists. With encouragement from the GA management, the Berkeley group made a commitment to assist GA in sharing the

burden of this analysis work and implementing improvements in the process. 
The ion temperature measurements at DIII-D are based on spectroscopic observations of the signal from the Charge Exchange Recombination (CER-process) when the neutral beams are present in the plasma. By now there are 32 chords ( 16 looking in vertically at the plasma, and 16 looking in the horizontal plane) looking at different locations in the plasma along the beam path. The spectral signal corresponding to each of the chords is analyzed separately, and the analysis is complicated because the measured spectrum has contributions not only from the CER-process with the beam, but also from other atomic processes near the edge of the plasma. The relative intensities of these components can vary greatly, and in unfavorable cases, the lines emitted at the edge can interfere severely with the temperature measurement at the beam location. The analysis is further complicated by the presence of fainter impurity lines from atomic transitions of mainly carbon, nickel and oxygen. Due to the complexity of the data, great care is required in the analysis and refits of the data are often required. Evaluation of the ion temperature fit is made between the iterations. This evaluation is based on experience that one gains only after relatively long hands-on experience with the analysis. The recent addition of new chords to obtain information at the edge of the plasma makes the procedure even more time consuming.

Considerable progress has been made along two lines. First, beginning with the assignment of one of our graduate students (D. Finkenthal) to San Diego for an extended period in the summer of 1989, the Berkeley group began to share the workload of reducing the ion temperature profile data by the labor-intensive methods then in place. Dr. Taina Kurki-Suonio, who joined our group as a postdoctoral fellow in May 1989, continued some of this work. This load-sharing enabled Dr. R. Groebner at GA, who had previously been almost entirely responsible for producing the profiles, to devote more time to improving the procedure. Secondly, a graduate student expert in computer programming (B. Modi) spent the summer of 1990 at GA, during which he assisted the GA group in implementing various planned improvements to the analysis code as well as some of his own ideas. Major contributions during the summer were to speed up the error analysis algorithm substantially and to examine the software carefully to make speed improvements where possible. He is now contributing toward the ultimate automation of the ion temperature profile analysis by installing a new least squares minimizing algorithm in the code. This work, when completed, will be the main topic of Mr. Modi's Master's Thesis.

\section{L-H TRANSITION}

Understanding why and how the H-mode exhibits improved particle and thermal confinement is essential before a commercial reactor can be designed. In close collaboration with Drs. Groebner and Burrell at GA, Dr. Kurki-Suonio has studied the details of thermal and angular momentum transport in DIII-D plasma across the L-H transition. Based on plasma profiles obtained from hot ion $\mathrm{H}$-mode discharges on DIII-D, the transport analysis showed that the improrement in confinement takes place all across the plasma cross-section (and not just at the edge). The improvement is not instantaneous. A drop in the diffusivities was observed to begin within $30 \mathrm{~ms}$ following the transition and the im- 
provement continues until at least $80 \mathrm{~ms}$ after the transition. The dominant improvement is in the electron channel. The nature of changes in the electron density is similar to the changes in diffusivities suggesting that the density gradient length is a critical quantity in the L-H transition. Experimental data is compared to a model for ITG mode. Stabilization of the ITG-mode does not seem to take place in the L-H transition, but the plasma might be marginally stable against the mode. The preliminary results were presented at the Division of Plasma Physics meeting, November 1990, and a complete report is now being prepared for publication in Nuclear Fusion.

\section{DIVERTOR}

A graduate student ( $Q$. Nguyen) has begun work on designs to reduce the divertor heat flux by employing poloidal coils to spread the scrape-off layer field lines over a wider area before they intersect the divertor plates. First results were presented at the Division of Plasma Physics meeting, Norember 1990, and a report has been prepared, co-authored with Drs. Stambaugh and Fowler.

The method appears promising and may contribute to improved confinement by providing design flexibility to simultaneously handle the heat flux together with appropriate baffling to contain and pump gases and impurities that would otherwise spoil the confinement.

\section{THEORY}

Two papers have been completed applying thermodynamic methods to calculate upper bounds on fluctuation levels and growth constants for electrostatic drift waves. The aim of this work was to try to understand the origin of the empirically-observed strong dependence of the global energy confinement time on the current, whereas the linear growth rates and diffusion estimates based on them depend mainly on the toroidal field (gyro-Bohm scaling). As was noted in our original grant proposal, this is a central issue in the design of future tokamaks (including BPX and ITER), especially in the strong reliance in these designs on elongation of the plasma cross-section as a means of increasing the current; and DIII-D, operating at high beta and an elongation $\kappa \geq 2$, is uniquely suited to exploring this issue.

At this writing, the origin of the current dependence is still not understood, either from theory or experimental analysis. However, the thermodynamic results, of wide validity even for complex geometries such as DIII-D, do offer a clue. Namely, the non-linear thermodynamic bound on electrostatic fluctuation levels can depend on the current, but the bound on the linear growth constant does not. This suggests looking for a direct correlation of fluctuation levels and the current, as well as correlation with the local thermal diffusion coefficient, $\chi$, as discussed below. The new thermodynamic theory, based on gyrokinetic equations, was carried out by a graduate student (D. Hua) in collaboration with the Principal Investigator and Dr. Alain Brizard, a postdoctoral fellow at LBL. This 
work will be a part of Mr. Hua's $\mathrm{PhD}$ thesis, soon to be completed.

\section{PROPOSED TASKS FY92-94}

Our main objective will continue to be providing support for DIII-D data analysis, together with developing and adapting theoretical models as needed. We expect to contribute in three areas, as follows

1. Continue to participate in transport analysis using the ONETWO transport code and in reduction of the experimental data required as input to this analysis.

2. Increase participation in data analysis relevant to edge plasma physics, including divertor experiments, and seek improvements in divertor design.

3. Contribute to improved theoretical and computational models required for the above, including: (a) Stability and transport calculations in DIII-D geometry, (b) Edge physics models taking into account particle orbits, non-Maxwellian distributions, and associated electric fields.

\section{TASKS FOR FY92}

In FY92, we propose to complete ongoing projects described above and undertake new tasks including the following:

1. As a continuation to our earlier analysis, we will continue to study the dynamics of confinement improvement after the L-H transition. Key data is the time-behavior of density fluctuations (obtained from a GA-UCLA collaboration) at different radial locations in the plasma following a transition from the L-mode to the H-mode. In particular, we want to compare the dynamics of the thermal diffusivities described above to the dynamics exhibited by turbulence fluctuations to see if turbulence suppression propagates inward at the same rate as the reduction in $\chi$ 's.

2. Adapt an existing linear stability code to calculate thresholds and growth rates for the ion temperature gradient (ITG) mode in DIII-D non-circular geometry. This entails parametrizing DIII-D flux surfaces obtained from the EFIT code as required for the stability code developed by Dr. Xuqiao Xu, formerly at UCSD and now a postdoctoral fellow in the Department of Electrical Engineering and Computer Science at Berkeley.

3. In collaboration with OR:VL and GA, analyze transport of helium ions in DIII-D and determine the ratio of helium concentration in the core plasma and the edge plasma. This has important implications for helium ash removal in ITER. Considerable data exists, not yet analyzed, as a result of helium discharge cleaning in DIII-D and more data can be provided if results warrant it.

4. Continue divertor design study in collaboration with GA and begin collaboration with Dr. Porter's DIII-D group on edge physics, with emphasis on accounting for discrepancies between observed divertor heat loads and those calculated by the B-2 code (2 to 4 times higher in some instances). Subjects for study include improved modelling of plasma 
sheaths at grazing incidence (finite orbit effects), effects of non-Maxwellian distributions, and characterization of energetic particle orbits near the separatrix.

5. As a continuation to earlier work done in the UCB-GA-LLNL collaboration, we plan to look at the dynamics possibly responsible for producing the flat, shouldered electron density profiles observed in DIII-D H-mode plasmas. In a previous transport study it has been shown that a significant inward pinch term was required to numerically reproduce the observed density profile. In this study the particle diffusion coefficient and the pinch velocity were empirically adjusted to obtain consistency between the calculated and experimental density profiles. We plan to take this study further by including theoretical models. In particular, we are looking at the thermoelectric-type effects as the source :or the inward pinch. Dr. Kurki-Suonio has already implemented a model (by R. Waltz) for a drift-wave produced thermal pinch into the ONETWO code and preliminary runs have indicated that the particle flux due to thermal pinch is a significant fraction of the total flux. The next step is to choose an appropriate set of discharges for analysis, and compare the experimental profiles to the ones obtained from the upgraded version of ONETWO, in which the value for the pinch velocity now comes from a theoretical model.

\section{PUBLICATIONS AND TALKS}

Publications (10)

1. K.H. Burrell, T. Kurki-Suonio et al., 'Comparison of Thermal and Angular Momentum Transport in Neutral Beam-Heated Hot-Ion H- and L-mode Discharges in DIII-D', Proceedings of the 17th European Conference on Controlled Fusion and Plasma Physics (European Physical Society, Petit-Lancy, Switzerland, 1990), Vol. 14B, Part I, p.271, and General Atomics Report GA-A2005S.

2. DIII-D Research Team (T. Kurki-Suonio as a UCB team member), 'Recent Results with Deuterium Plasma Operation of the DIII-D Tolamak', to be published in J. of Plasma Physics and Controlled Fusion.

3. J.L. Luxon et al. (T. Kurki-Suonio as a UCB team member), 'Recent Results from DIII-D and Their Implications for Next Generation Tokamaks', Proceedings of the 17th European Conference on Controlled Fusion and Plasma Physics (European Physical Society, Petit-Lancy, Switzerland, 1990).

4. M.A. Mahdavi et al. (T. Kurki-Suonio as a UCB team member), 'Plasma Boundary Experiments on DIII-D Tokamak', Proceedings of the 9th International Conference on Plasma Surface Interactions in Controlled Fusion Devices, May 20-25, 1990, in Bournemouth, United Kingdom, and General Atomics report GA-A20130 (1990).

5. D.P. Schissel et al. (T. Kurki-Suonio as a UCB team member), 'The Effects of Carbonization on the Confinement Properties of the DIII-D H-Mode', Proceedings of the 17th European Conference on Controlled Fusion and Plasma Physics (European Physical 
Society, Petit-Lancy, Switzerland, 1990), Vol. 14B, Part I, p.275.

6. K.H. Burrell, T. Kurki-Suonio et al., 'Transport in Auxiliary-Heated, Hot-Ion H-Mode and L-Mode Discharges in the DIII-D Tokamak', General Atomics Report GAA20277, and to be published in Plasma Physics and Controlled Nuclear Fusion Research 1990, (IAEA, Washington, DC, 1990).

7. DIII-D Team (T. Kurki-Suonio as a UCB team member), 'DIII-D Research Program Progress', to be published in Plasma Physics and Controlled Nuclear Fusion Research 1990. (IAEA, Washington, DC, 1990).

8. T.K. Fowler, and P.J. Morrison, 'Extrerıal Bounds on Drift Wave Growth Rates and Transport', UC-BFE-009, March 20, 1990, and submitted for publication in Nuclear Fusion.

9. A. Brizard, D. Hua, and T.K. Fowler," 'Gyrokinetic Free Energy in Tokamak Plasmas', UC-BFE-022, March 7, 1991, and submitted for publication in Nuclear Fusion.

10. T.K. Kurki-Suonio, R.J. Groebner, and K.H. Burrell, 'Changes in Local Confinement after L-H Transition on DIII-D', General Atomics Report GA-A20450, and to be submitted to Nuclear Fusion.

\section{Reports (6)}

11. T. Kurki-Suonio and R. Groebner, 'Effect of ELMs on Ion Temperature Profile', DIII-D Physics Memo, D3DPM No. 9004

12. D. Finkenthal, 'A Cookbook Guide to Learning The General Atomics CERFIT Code for Ion Temperature Analysis', UC-BFE-012, April 4, 1990.

13. Z. Covaliu, 'Sensitivity of a Tokamak Heat Transport Model to Edge Effects and Density profile', UC-BFE-013, June 18, 1990.

14. B. Modi, 'Speeding Up the CERFIT Code for Ion Temperature Analysis at DIII-D Tokamak', UC-BFE-019, 1991.

15. Q. Nguyen T.K. Fowler, and R.D. Stambaugh, 'Divertor Heat Flux Reduction Using Poloidal Coils', UC-BFE-020, 1991.

16. A. Brizard, 'Thermodynamic Analysis of Unstable Gyrokinetic Plasmas', UCBFE-021, 1991.

$$
\text { Talks, Posters (8) }
$$

17. T.K. Fowler, 'Thermodynamic Insights into Tokamak Confinement', poster at the Division of Plasma Physics meeting, APS, Hollywood, Flotida, November 1988.

18. T.K. Fowler, and P.J. Morrison, 'Bounds on Instability Growth Rates in Toka-

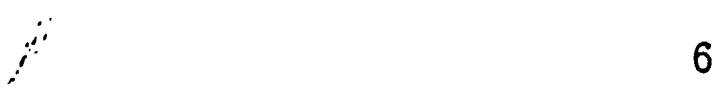


maks', Sherwood Theory Meeting, San Antonio, March 1989.

19. P.J. Morrison, and T.K. Fowler, 'Extremal Bounds on Instability Growth Rates', poster at the Division of Plasma Physics meeting, APS, Anaheim, California, November 1989.

20. G.D. Porter, D. Hill, M. Perry, and T. Kurki-Suonio, 'Transient Analysis of Particle Transport in DIII-D H-Mode Plasmas, (A talk given in The Transport Task Force Meeting, Hilton Head, South Carolina, Feb 17-23 1990)

21. D.F. Finkenthal, and T.K. Fowler, 'Effect of the TAE mode on Achieving Ignition in Tokamak Reactors', poster at the Division of Plasma Physics meeting, APS, Cincinnati, Ohio, 1990.

22. D. Hua, A. Brizard, and T.K. Fowler, 'Gyrokinetic Free Energy in Tokamak Plasmas, poster at the Division of Plasma Physics meeting, APS, Cincinnati, Ohio, 1990.

23. Q. Nguyen, T.K. Fowler, and R.D. Stambaugh, 'Flux Expansion at the Divertor Plates', poster at the Division of Plasma Physics meeting, APS, Cincinnati, Ohio, 1990.

24. T. Kurki-Suonio and R. Groebner, 'Changes in Local Confinement after an L-H Transition in DIII-D', poster at the Division of Plasma Physics meeting, APS, Cincinnati, Ohio, 1990.

\section{ORGANIZATION}

Principal Investigator

Postdoctoral Fellow

Four(4) graduate students
T. Kenneth Fowler

Taina Kurki-Suonio

In addition to the above participants, beginning with the fall 1989 semester we established a Transport Seminar that meets regularly with participation from the UCB Physics Department, the UCB Department of Electrical Engineering and Computer Science, LBL and LLNL. Dr. Gary Porter, head of the Livermore DIII-D team, participates in these meetings. Principal contacts at General Atomics have been Drs. Stambaugh, Burrell, and Groebner. We intend to maintain the ongoing collaboration with $\mathrm{Dr}$. Stambaugh's group, now reorganized to focus on Boundary Physics and Technology. We will also maintain contact with other parts of the DIII-D organization, under the overall leadership of Dr. Simonen, and contribute wherever appropriate.

\section{FUNDING}

The budget request is attached. Funding provides support for 4 graduate students 
(the current number), one postdoctoral fellow, and two months summer salary for Professor Fowler. The request also includes funds for travel to and from San Diego consistent with actual experience under the present grant, and foreign travel funds for one person to attend the IAEA conferences in 1992 and 1994. Note that the request for graduate student support now includes a provision to pay tuition fees, in an anticipated change in University policy effective January, 1992.

\title{
DISCLAIMER
}

\begin{abstract}
This report was prepared as an account of work sponsored by an agency of the United States Government. Neither the United States Covernment nor any agency thereof, nor any of their employees, makes any warranty, express or implied, or assumes any legal liability or responsibility for the accuracy, completeness, or usefulness of any information, apparatus, product, or process disclosed, or represents that its use would not infringe privately owned rights. Referenc; herein to any specific commercial product, process, or service by trade name, trademark, manufacturer, or otherwisi does not necessarily constitute or imply its endorsement, recommendation, or favoring by the United States Government or any agency thereof. The views and opinions of authors expressed herein do not necessarily state or reflect those of the United States Government or any agency thereof.
\end{abstract}



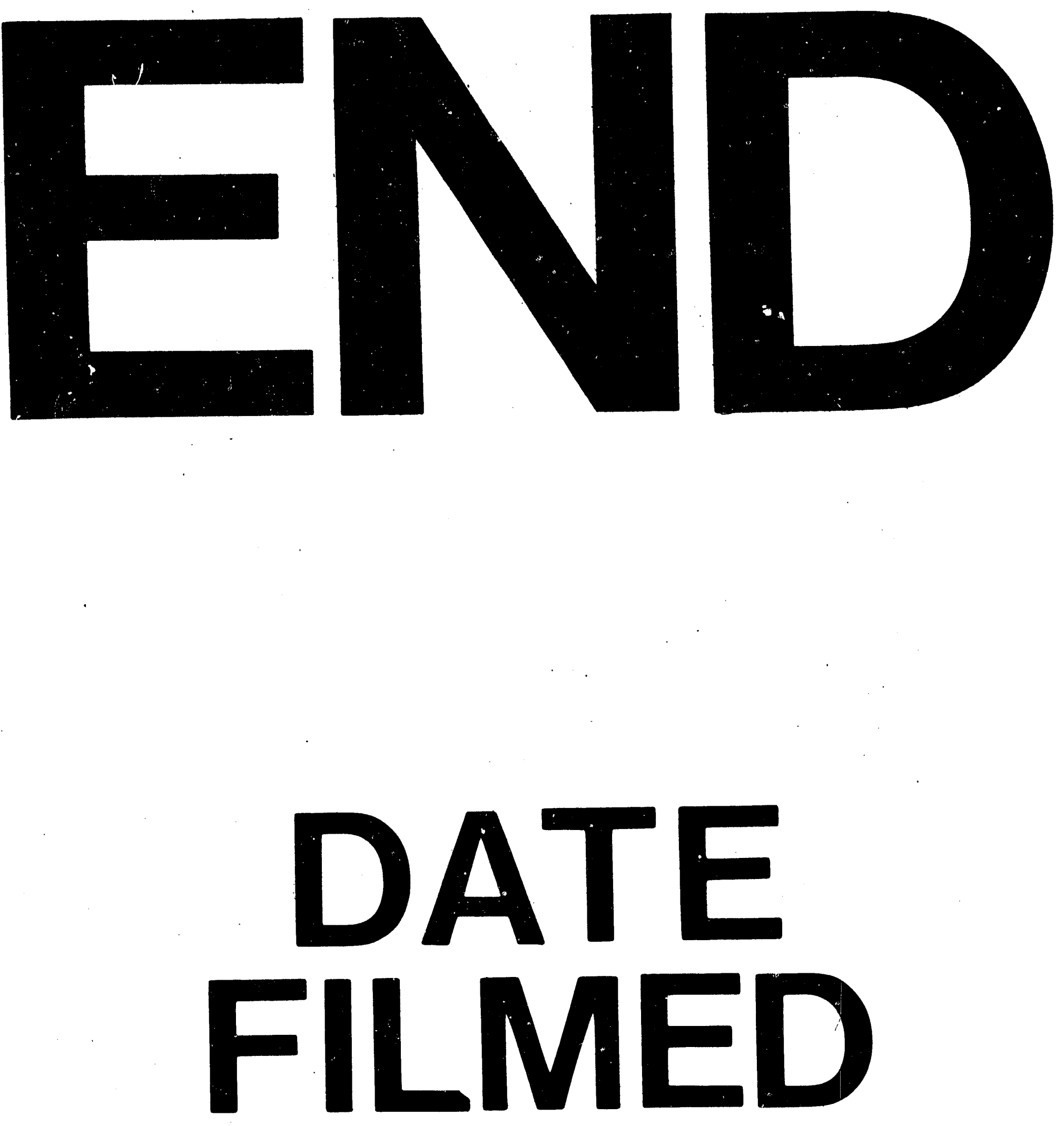

1

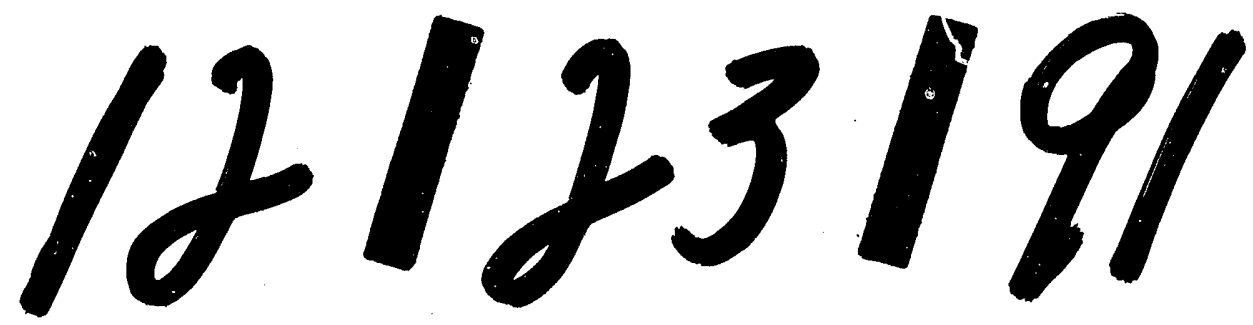

7 
